\title{
TRACKING HUMAN UPPER-LIMB MOVEMENTS WITH SLIDING MODE CONTROL TYPE-II FUZZY
}

\section{LOGIC}

\author{
Siti Khadijah Ali \\ Department of Automatic Control \\ and System Engineering \\ University of Sheffield \\ Skali1@shef.ac.uk
}

\author{
Ahmad Riyad Firdaus \\ Department of Automatic Control \\ and System Engineering \\ University of Sheffield \\ arfirdaus2@shef.ac.uk
}

\author{
*M.O.Tokhi \\ Department of Automatic Control \\ and System Engineering \\ University of Sheffield \\ o.tokhi@shef.ac.uk
}

Ghasaq Al-Rezage Department of Automatic Control and System Engineering University of Sheffield GSmousa1@sheffield.ac.uk

\begin{abstract}
A knowledge of human upper-limb structure and its mechanical functions are important for developing an exoskeleton. The Sliding Mode Control with Fuzzy Type-II is proposed to control the movements of the human extremity joints. The Lagrange method is used to model the dynamics system of human upper-limb. The findings demonstrate the effectiveness of the proposed controller in tracking the desired motion and it is also able to eliminate the chattering problem as well as deal with uncertainties.
\end{abstract}

Keywords-Upper-extremity, sliding mode control, type-II fuzzy logic, robust controller

\section{INTRODUCTION}

Muscle fatigue is a common phenomenon of performance degradation in humans. There are various factors that lead to muscle fatigue. These include manual handling of objects, heavy physical activities, repetitive work, improper body posture and duration of exposure to working environment [1]. Muscle fatigue could affect human's quality of life and there is high risk of getting musculoskeletal disease (MSD) and performance degradation. As reported by Martinez et al. (2008) and Health and Safety Executive (2002), MSD is the most common disease that is experienced by industrial workers in Europe. Meanwhile, 25\% of workers suffer from backache and $23 \%$ are reported having muscular pain [2].

Rehabilitation procedure and industrial working environment also know to lead to muscle fatigue. Ambrosini et al. (2014) and Lalitharatne et al. (2012) have reported that users or patients have high probability to get fatigued during rehabilitation process. Domestic activities such as painting and fixing a bulb might trigger the muscles to fatigue, same as activities in industry and during rehabilitation process [5].

These situations have motivated the development of robotic exoskeletons as a tool or solution to solve muscle fatigue or MSD [6]. However, it is important to note that, the developed exoskeleton should mimics the human upper-limb movements. This is to ensure the safety for the human. In addition, there are other elements that need to be considered for developing an exoskeleton: the design, the controller, the sensor and the actuator. The aim of this paper is to develop a suitable controller for an upper limb exoskeleton. In general, there are two categories of the existing control approach: linear and nonlinear control approach. The examples of linear control approach are PID admittance control and PD [7], [8]. However, these type of controllers have limitation in regard to the movement of the human upper-limb. This is due to the nature of the human upper-limb; non-linear. Hence, this drawback has motivated the development of non-liner control strategies such as computed torque control, nonlinear PID and other related mechanisms.

The sliding mode control (SMC) is a model-based control methods, is one of the example of a non-linear control approach. This controller has been applied to many applications such as UAVs as well as in developing an exoskeleton [9]-[12]. The advantages of the SMC are the structure is simple and fast to response. In addition, SMC also has an ability to ensure trajectory tracking performance in the presence of system perturbation, external disturbance and parametric uncertainties [13]. However, the high control activities or known as chattering has limit the capability for the SMC in practical application. The chattering occur due to the discontinuous sign function.

One way to solve this limitation is by replacing the sign function with boundary layer [14], sat function [15] or fuzzy logic system [9], [16]-[18]. These approaches show that the chattering could be eliminated/reduced. In terms of tracking performance, however, these approaches show a negative affect and also cause an increase in a steady state error [15]. In this work, therefore a hybrid mechanism of SMC with fuzzy type-II is explored to control the high non-linear motion of human upper-limb. The results obtained from this work could provide an information to develop controller for an upper-limb exoskeleton in future.

\section{Proposed APPROACH}

An upper-limb exoskeleton is developed and attached to humans' upper-limb. Figure II.1 shows the exoskeleton with six degree-of-freedom (DOF) attached to human upper extremity. As mentioned in previous section, the current paper is aim to investigate the potential of the sliding mode control with type-II fuzzy logic in controlling the movements of the upper-limb. Therefore, in this work, only one DOF for each joint of human upper-limb, will be used to examine the performance of the controller, and the joints namely: shoulder and elbow joints. The parameters used in this work were taken from [19] and the human design is from [20]. 


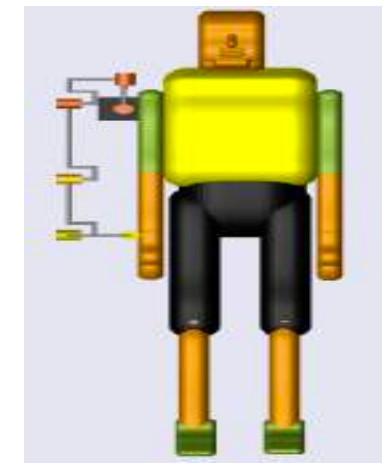

Figure II.1 Human with exoskeleton's joints

In the next section, the dynamic model for two links of human is presented.

\section{A. Dynamic Model}

Generally, the dynamic model is developed to build the relationship between the desired trajectory and the input torque of the two links. In addition, the dynamic model is very important for model-based control approach [16]. Thus, the sliding mode control is proposed based on the dynamic of the human upper-limb.

In this work, the Lagrange method is used to develop analytical dynamic model of the human arm. However, three assumptions are made for simplification purposes: (1) The wrist joint is assumed to be fixed. (2) The hand is assumed to be part of lower arm. (3) This model could be classified as conservative model because the frictional elements acting on the human joints is ignored. Using the Lagrange method with system's kinetic and potential energies, the general form of dynamic equation for the human joints can be expressed as:

$\mathrm{M}_{\mathrm{h}}\left(\theta_{\mathrm{h}}\right) \ddot{\theta}+\mathrm{C}_{\mathrm{h}}\left(\theta_{\mathrm{h}}, \dot{\theta}_{\mathrm{h}}\right) \dot{\theta}_{\mathrm{h}}+\mathrm{G}_{\mathrm{h}}\left(\theta_{\mathrm{h}}\right)=\tau_{\mathrm{h}}$

where, $\mathrm{M}_{h}, \mathrm{C}_{\mathrm{h}}, \mathrm{G}_{\mathrm{h}}$ and $\tau_{\mathrm{h}}$ are the moment of inertia, coriolis and centrifugal matrix, gravity vector and input torques. The angles for shoulder and elbow joints are represented as $\theta_{\mathrm{h}}=\left[\theta_{1}, \theta_{2}\right]^{\mathrm{T}}$ and the input torques $\tau_{\mathrm{h}}=\left[\tau_{1}, \tau_{2}\right]^{\mathrm{T}}$. Equation (1) can be rewritten for joint 1 and joint 2 as follows:

$\ddot{\theta}_{1}=\frac{a_{22}}{A}\left[\tau_{1}-\left(b_{11} \dot{\theta}_{1}+b_{12} \dot{\theta}_{2}\right)-g_{1}\right]-\frac{a_{12}}{A}\left[\tau_{2}-\left(b_{21} \dot{\theta}_{1}+b_{22} \dot{\theta}_{2}\right)-g_{2}\right]$ $\ddot{\theta}_{2}=-\frac{a_{21}}{A}\left[\tau_{1}-\left(b_{11} \dot{\theta}_{1}+b_{12} \dot{\theta}_{2}\right)-g_{1}\right]+\frac{a_{11}}{A}\left[\tau_{2}-\left(b_{21} \dot{\theta}_{1}+b_{22} \dot{\theta}_{2}\right)-g_{2}\right]$

With the corresponding terms:

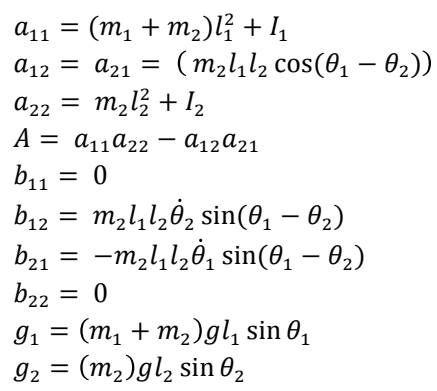

The proposed control system is presented in the next section.

\section{B. Control system approach}

A hybrid mechanism of sliding mode control (SMC) and interval Type-2 fuzzy logic system (IT2FLS) is proposed. Three stages of control design were conducted to achieve the desired performance of the exoskeleton.

\section{1) Sliding surface design}

The general form of sliding surface can be represented as:

$\sigma(x, t)=S x(t)$

where $\mathrm{S}$ is a matrix of positive constant elements with dimension $\mathrm{m} \times \mathrm{n}$. For second order systems, the scalar form of sliding surface can be defined as [9]:

$\sigma(t)=s e+\dot{e}$

The variable $s$ is definitely positive constant whereas e and $\dot{e}$ are error and change of error of system response respectively. Hence, the sliding manifolds of the upper limb exoskeleton system are represented as:

$\sigma_{1}=s_{1}\left(\theta_{1}-\theta_{1 d}\right)+\left(\dot{\theta}_{1}-\dot{\theta}_{1 d}\right)$

$\sigma_{2}=s_{2}\left(\theta_{2}-\theta_{2 d}\right)+\left(\dot{\theta}_{2}-\dot{\theta}_{2 d}\right)$

Therefore, the sliding surface matrix can expressed as:

$\sigma=\left[\begin{array}{cccc}s_{1} & 1 & 0 & 0 \\ 0 & 0 & s_{2} & 1\end{array}\right] x+\left[\begin{array}{l}-s_{1} \theta_{1 d} \\ -s_{2} \theta_{2 d}\end{array}\right]$

where $\sigma_{1}$ and $\sigma_{2}$ denote sliding surface for joint 1 and joint 2 respectively, whereas $\theta_{1 \mathrm{~d}}$ and $\theta_{2 \mathrm{~d}}$ represent the desired position for joint 1 and joint 2 respectively.

\section{2) Sliding mode control design}

The equation $\ddot{\theta}_{2}=-\frac{a_{21}}{A}\left[\tau_{1}-\left(b_{11} \dot{\theta}_{1}+b_{12} \dot{\theta}_{2}\right)-g_{1}\right]+\frac{a_{11}}{A}\left[\tau_{2}-\right.$ $\left.\left(b_{21} \dot{\theta}_{1}+b_{22} \dot{\theta}_{2}\right)-g_{2}\right]$

( 2 can be represent in general, as follows :

$\ddot{\theta}=M_{h}\left(\theta_{h}\right)^{-1}\left[\tau_{h}-C_{h}\left(\theta_{h}, \dot{\theta}_{h}\right) \dot{\theta}_{h}-G_{h}\left(\theta_{h}\right)\right]$

For the simplicity purpose, equation (8) could be written in state-space representation as in follow:

$\dot{x}(t)=f(x, t)+B(x, t) u(t)$

where the state vectors are defined as $\mathrm{x}_{1}=\theta_{1} ; \mathrm{x}_{2}=\dot{\theta}_{1} ; \mathrm{x}_{3}=$ $\theta_{2} ; x_{4}=\dot{\theta}_{2}$. Therefore, from (2) and (9), the vectors $f(x, t)$ and $\mathrm{B}(\mathrm{x}, \mathrm{t})$ can be expressed as: 
$f(x)=\left[\begin{array}{c}\frac{a_{22}}{A}\left[-\left(b_{11} x_{2}+b_{12} x_{4}\right)-g_{1}\right]-\frac{a_{12}}{A}\left[-\left(b_{21} x_{2}+b_{22} x_{4}\right)-g_{2}\right] \\ x_{4} \\ -\frac{a_{21}}{A}\left[-\left(b_{11} x_{2}+b_{12} x_{4}\right)-g_{1}\right]+\frac{a_{11}}{A}\left[-\left(b_{21} x_{2}+b_{22} x_{4}\right)-g_{2}\right]\end{array}\right]$

$B(x)=\left[\begin{array}{cc}0 & 0 \\ \frac{a_{22}}{A} & -\frac{a_{12}}{A} \\ 0 & 0 \\ -\frac{a_{21}}{A} & \frac{a_{11}}{A}\end{array}\right]$

As mentioned in Section II.A, the SMC is model-based control approach. Therefore, it is important to have the best estimation model as possible to the real plant. The estimation model in this work, could be acquired from equation (8) and is presented as below:

$\ddot{\theta}=\widehat{M}_{h}\left(\theta_{h}\right)^{-1}\left[\tau_{h}-\widehat{C}_{h}\left(\theta_{h}, \dot{\theta}_{h}\right) \dot{\theta}_{h}-\widehat{G}_{h}\left(\theta_{h}\right)\right]$

where the symbol represents the estimation model of the system. Since the estimation model is gathered from equation (8), thus $\hat{\mathrm{f}}(\mathrm{x})$ and $\widehat{\mathrm{B}}(\mathrm{x})$ are as below :

$\hat{f}(x)=f(x)\left[\begin{array}{c}\frac{x_{22}}{A}\left[-\left(b_{11} x_{2}+b_{12} x_{4}\right)-g_{1}\right]-\frac{a_{12}}{A}\left[-\left(b_{21} x_{2}+b_{22} x_{4}\right)-g_{2}\right] \\ x_{4} \\ -\frac{a_{21}}{A}\left[-\left(b_{11} x_{2}+b_{12} x_{4}\right)-g_{1}\right]+\frac{a_{11}}{A}\left[-\left(b_{21} x_{2}+b_{22} x_{4}\right)-g_{2}\right]\end{array}\right]$

$\hat{B}(x)=\left[\begin{array}{cc}0 & 0 \\ \frac{a_{22}}{A} & -\frac{a_{12}}{A} \\ 0 & 0 \\ -\frac{a_{21}}{A} & \frac{a_{11}}{A}\end{array}\right]$

Theoretically, in SMC, the control law $(\mathrm{u})$ is constructed by two signals, namely equivalent control $\left(u_{e q}\right)$, and switching control $\left(u_{s w}\right)$ as:

$u=u_{e q}+u_{s w}$

The sliding mode will occur if and only if $\sigma(\mathrm{x}, \mathrm{t})=$ 0 and $\dot{\sigma}(\mathrm{x}, \mathrm{t})=0$. Therefore, by using equations (3), (9), and (13) the equivalent control (ueq) can be defined as [9]:

$u_{e q}=-(S \hat{B}(x, t))^{-1} S \hat{f}(x, t)$

Furthermore, considering the sliding condition $\sigma^{T} \dot{\sigma}=$ $\sigma\left(S \hat{B} u_{n}\right)=\sigma u_{n}^{*}<0$, the switching control $\left(u_{s w}\right)$ is expressed as [9]:

$\mathrm{u}_{\mathrm{sw}}=-\mathrm{k}(\mathrm{S} \widehat{\mathrm{B}}(\mathrm{x}, \mathrm{t}))^{-1} \operatorname{sign}(\sigma) ;$ if $(\mathrm{SB})$ invertible

3) Sliding mode control-based interval type-II fuzzy logic

The general structure of the proposed method is depicted as [9]:

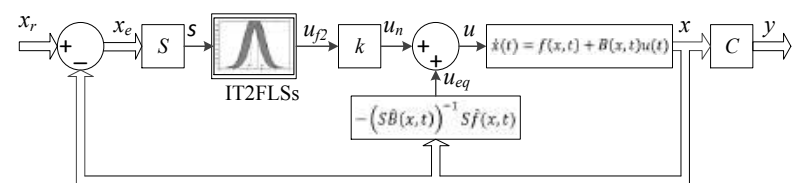

Figure II.2 SMC-based IT2FLC architecture

The fuzzy membership functions (MFs) for input is constructed by the sliding surface $(\sigma)$ values as depicted in

Figure II. 3 and for output in

Figure II.4. Each of these has 7 membership functions, namely: Negative Big (NB), Negative Medium (NM), Negative Small (NS), Zero (Z), Positive Small (PS), Positive Medium (PM), and Positive Big (PB).

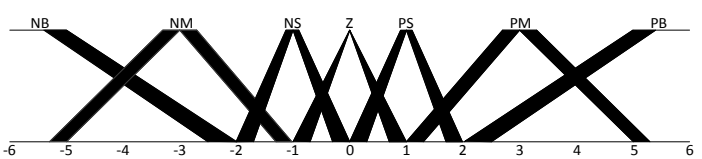

Figure II.3 Sliding surface $(\sigma) \mathrm{MFs}$

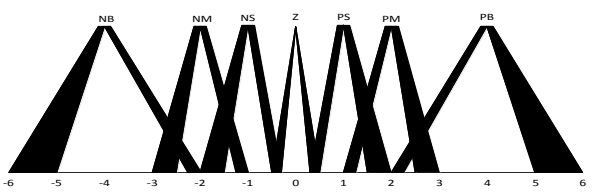

Figure II.4 Fuzzy output MFs

Furthermore, from the fuzzy input and output, the desired switching control $\left(u_{s w}\right)$ can be set up by developing the fuzzy rules-base as in Table 1. Therefore, the switching control can be defined as:

$u_{s w}=k u_{f 2}$

TABLE 1 THE RULES OF IT2FLC-SMC

\begin{tabular}{|l|c|c|c|c|c|c|c|}
\hline & \multicolumn{7}{|c|}{$\sigma$ (Sliding mode) } \\
\cline { 2 - 8 } & NB & NM & NS & Z & PS & PM & PB \\
\hline Out $_{\left(\text {fuzzy }_{2}\right)}$ & PB & PM & PS & Z & NS & NM & NB \\
\hline
\end{tabular}

where $k$ is a constant to achieve the desired trajectory responses, while $u_{f 2}$ denotes the crisp values of IT2FLC. Thus, the equations (11) and (13) are used to produce the overall control signal of SMC as:

$u=-(S \hat{B}(x, t))^{-1} S \hat{f}(x, t)+k u_{f 2}$

\section{EXPERIMENTS AND RESULTS}

In order to evaluate the performance of the SMC with typeII fuzzy logic algorithm presented in the previous section, preliminary tracking experiments were carried out. As mentioned above, the measurements of the lengths and the mass of the upper-arm and lower-arm as well as the inertia are taken from [13].

The tracking experiments are conducted on the flexion/extension rotation of shoulder and elbow. For the first 
experiment, the tracking performance of SMC with type-II fuzzy logic are compared to SMC with conventional fuzzy algorithm and conventional PID control system. The PID is chosen as it is most use in industrial application. The gain for $\mathrm{P}$, $\mathrm{I}$ and $\mathrm{D}$ are acquired by try-and-error. For the second experiment, the tracking performance was compared with conventional fuzzy algorithm. Similar as P, I and D, gains for k, $\omega_{1}$ and $\omega_{2}$ are also obtained by try-and-error.

The proposed comparative position tracking tests were started with $\pi / 2$ with a nine-seconds-long trajectory. This is to examine how good the controller can work in order to ensure that it can achieve the desired trajectory. Then, the second desired tracking position was with the amplitude of 0 degree for the first two-seconds, and 90 degrees for the next two-seconds, and then down to 0 until ninth-second. The predefined trajectory was thus:

$\theta_{d}=\left\{\begin{array}{lc}0 & 0 \leq t \leq 2 \\ \frac{p i}{2} & 2 \leq t \leq 4 \\ 0 & 4 \leq t \leq 9\end{array}\right.$

TABLE 2 PARAMETERS USED IN SMCFT1 AND SMCFT2

\begin{tabular}{|l|l|l|l|l|}
\hline $\begin{array}{l}\text { Reference } \\
\text { trajectory }\end{array}$ & Method & $\mathrm{k}$ & $\lambda_{1}$ & $\lambda_{2}$ \\
\hline $\begin{array}{l}\text { First } \\
\text { Experiment }\end{array}$ & SMCFT1 & 300 & 300 & 500 \\
\cline { 2 - 5 } & SMCFT2 & 4 & 6 & 5 \\
\hline $\begin{array}{l}\text { Second } \\
\text { experiment }\end{array}$ & SMCFT1 & 400 & 80 & 100 \\
\cline { 2 - 5 } & SMCFT2 & 4 & 6 & 10 \\
\hline
\end{tabular}

TABLE 3 PARAMETERS USED IN CONVENTIONAL PID

\begin{tabular}{|l|l|l|l|l|}
\hline $\begin{array}{l}\text { Reference } \\
\text { trajectory }\end{array}$ & Method & $\mathrm{P}$ & $\mathrm{I}$ & $\mathrm{D}$ \\
\hline $\begin{array}{l}\text { First } \\
\text { Experiment }\end{array}$ & Joint 1 & 1 & 1.3 & 0.5 \\
\cline { 2 - 5 } & Joint 2 & 0.9 & 1.3 & 0.5 \\
\hline
\end{tabular}

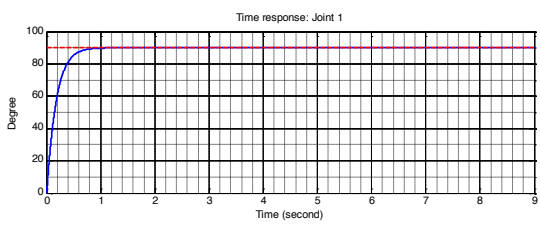

Time ressonse: Joint 2

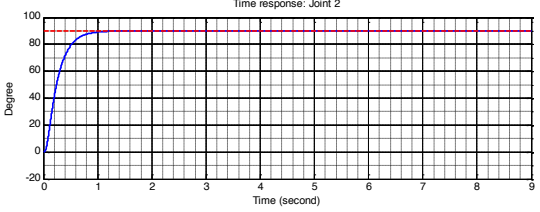

(a)

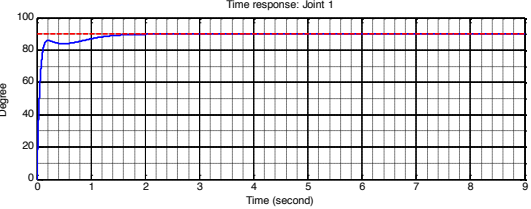

Time response: Joint 2

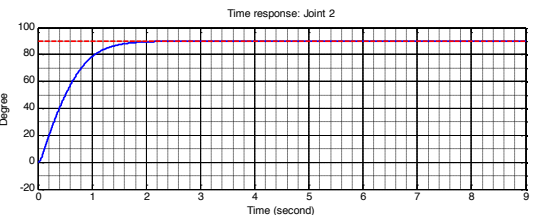

(b)
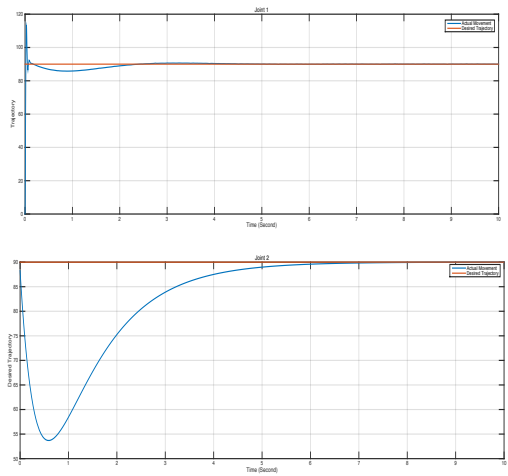

(c)

Figure III.1 Results of $\frac{\pi}{2}$ by using SMC with: (a) Type-II (b) Type-I fuzzy logic algorithm (c) Conventional PID controller

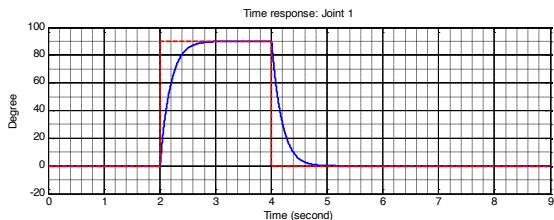

Thesents

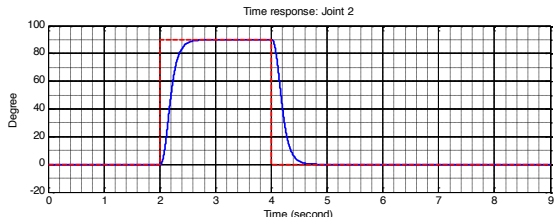

(a)
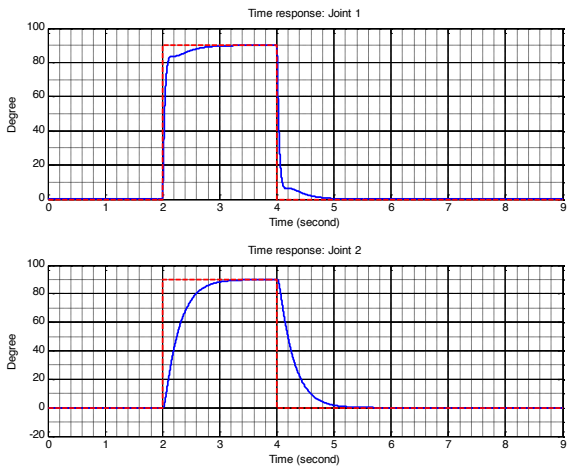

(b) 
Figure III.2 Results of second experiments by using SMC with: (a) Type-II (b) Type-I fuzzy logic algorithm

The trajectory tracking results are demonstrated in Figure III.1 and Figure III.2. As can be observed, the tracking performance of the SMC with type-II fuzzy logic algorithm was better than SMC with conventional fuzzy logic algorithm as well as conventional PID controller. The results also show that the former algorithm achieved better transient response compared to the latter algorithm. The chattering problem was eliminated with both algorithms. However, as noted in Figure III.1 (b), there is small chattering occur in the first joint when the joint was moving to desired trajectory. In contrast as noted in Figure III.1 (a), the response of first joint was smooth with the type-II fuzzy logic control. Figure III.2 also presents similar results as Figure III.1 and shows that the SMC with type-II fuzzy logic controller was able to control the movement to the desired trajectory.

TABLE 4 UPPER-LIMB PARAMETERS

\begin{tabular}{|l|l|l|l|}
\hline Variables & \multicolumn{2}{|l|}{ Values } & Units \\
\hline & Estimation & Plant & \\
\hline Mass upper-arm & 2.70 & 2.821 & $\mathrm{Kg}$ \\
\hline Mass lower-arm & 3.30 & 4.29 & $\mathrm{Kg}$ \\
\hline Length upper-arm & 0.3132 & 0.40716 & $\mathrm{~m}$ \\
\hline Length lower-arm & 0.2808 & 0.36504 & $\mathrm{~m}$ \\
\hline $\mathrm{I}_{\mathrm{zz} 1}$ & 0.00575 & 0.007475 & $\mathrm{Kg} \mathrm{m} 2$ \\
\hline $\mathrm{I}_{\mathrm{zz} 2}$ & 0.00313 & 0.004069 & $\mathrm{Kg} \mathrm{m} 2$ \\
\hline
\end{tabular}

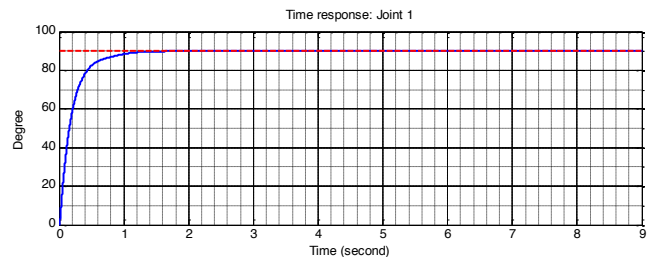

Time response: Joint 2

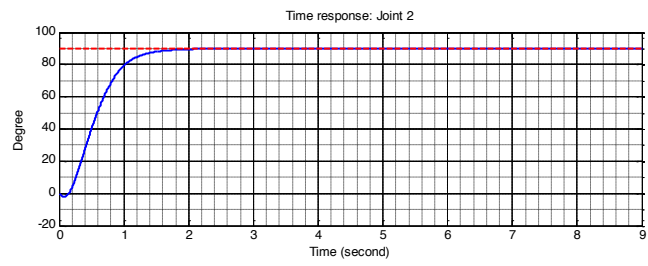

Figure III.3 Results of uncertainty

Table 3 shows the parameters used in this work together with mismatch values of each parameter. This is to show the robustness of the controller in which, as shown in Figure III.2, the system was still stable even with the values of the parameters for the estimation model mismatched by $30 \%$. Therefore, it is confirmed that this controller is suitable to be applied to exoskeleton for position control.

\section{CONCLUSION}

An investigation into application of SMC with type-II fuzzy algorithm in positioning control of human upper-limb has been presented. SMC has been employed due its robust performance with nonlinear dynamic systems and type-II fuzzy logic is used in order to eliminate the chattering problem that usually occurs in SMC. The results show that the controller is able to track the desired movement smoothly and without the chattering. Moreover, the controller is able to deal with uncertainties and hence is suitable for controlling the highly nonlinear human upper-limb system. In future, the proposed controller will be used to control the human model with frictional element. This element is included in order to make the human model realistic. The proposed control algorithm also be tested to control the exoskeleton without the need to measure muscular activation levels.

\section{ACKNOWLEDGMENT}

Siti Khadijah Ali, Ahmad Riyad Firdaus and Ghasaq AlRezage are on study leave supported by Malaysia of Higher Education and Universiti Putra Malaysia; and Indonesia Endowment Fund for Education, Ministry of Finance of Republic of Indonesia and Politeknik Negeri Batam Indonesia, and Higher Committee Education Development Iraq, respectively.

\section{REFERENCES}

[1] C. T. Coffin, "The use of a vertical arm support device to reduce upper extremity muscle firing in sonographers.," Work, vol. 42, no. 3, pp. 367-71, Jan. 2012.

[2] F. Martinez, I. Retolaza, a. Pujana-Arrese, a. Cenitagoya, J. Basurko, and J. Landaluze, "Design of a five actuated DoF upper limb exoskeleton oriented to workplace help," 2008 2nd IEEE RAS EMBS Int. Conf. Biomed. Robot. Biomechatronics, pp. 169-174, Oct. 2008.

[3] E. Ambrosini, S. Ferrante, M. Rossini, F. Molteni, M. Gföhler, W. Reichenfelser, A. Duschau-Wicke, G. Ferrigno, and A. Pedrocchi, "Functional and usability assessment of a robotic exoskeleton arm to support activities of daily life," Robotica, vol. 32, no. 2014, pp. 1213-1224, 2014.

[4] T. D. Lalitharatne, Y. Hayashi, K. Teramoto, and K. Kiguchi, "A study on effects of muscle fatigue on EMG-based control for human upper-limb powerassist," 2012 IEEE 6th Int. Conf. Inf. Autom. Sustain., pp. 124-128, Sep. 2012.

[5] I. L. Nunes and P. M. Bush, "Work-Related Musculoskeletal Disorders Assessment and Prevention," Ergon. Syst. Approach, pp. 1-31, 2011.

[6] M. G. Carmichael, D. Liu, and K. J. Waldron, "Investigation of reducing fatigue and musculoskeletal disorder with passive actuators," in 2010 IEEE/RSJ International Conference on Intelligent Robots and Systems, 2010, pp. 2481-2486.

[7] P. R. Culmer, A. E. Jackson, S. Makower, R. Richardson, J. A. Cozens, M. C. Levesley, and B. B. Bhakta, "A Control Strategy for Upper Limb Robotic 
Rehabilitation With a Dual Robot System," IEEE/ASME Trans. Mechatronics, vol. 15, no. 4, pp. 575-585, Aug. 2010.

[8] R. Kelly, "PD Control with Desired Gravity Compensation of Robotic Manipulators: A Review," Int. J. Rob. Res., vol. 16, no. 5, pp. 660-672, 1997.

[9] A. R. Firdaus and M. O. Tokhi, "Sliding mode control - Based interval type-II fuzzy logic controller for quadcopter UAVs," in 18th International Conference on Climbing and Walking Robots, 2015, pp. 1-8.

[10] S. Jezernik, R. G. V Wassink, and T. Keller, "Sliding mode closed-loop control of FES: controlling the shank movement," IEEE Trans. Biomed. ENGINNERING, vol. 51, no. 12, pp. 263-272, 2004.

[11] C.-T. Chang, H.-P. Huang, and J.-Y. Kuan, "Independent Joint Sliding Mode Control of a Humanoid Robot Arm," in IEEE Indutrial Electronics Society (IECON), 2007, pp. 2754-2759.

[12] M. H. Rahman, T. Kittel-Ouimet, M. Saad, J.-P. Kenne, and P. S. Archambault, "Development and control of a robotic exoskeleton for shoulder, elbow and forearm movement assistance," Appl. Bionics Biomech., vol. 9, no. 3, pp. 275-292, 2012.

[13] M. H. Rahman, M. Saad, J. P. Kenné, and P. S. Archambault, "Nonlinear Sliding Mode Control Implementation of an Upper Limb Exoskeleton Robot to Provide Passive Rehabilitation Therapy," in 5th International Conference on Intelligent Robotics and Applications, 2012, pp. 56-62.

[14] J.-J. E. Slotine and W. Li, Applied Nonlinear Control, vol. 62, no. 7. 1991.

[15] M. H. Rahman, M. Saad, J.-P. Kenné, and P. S. Archambault, "Control of an exoskeleton robot arm with sliding mode exponential reaching law," Int. J. Control. Autom. Syst., vol. 11, no. 1, pp. 92-104, 2013.

[16] Q. Liu, D. Liu, W. Meng, Z. Zhou, and Q. Ai, "Fuzzy Sliding Mode Control of a Multi-DOF Parallel Robot in Rehabilitation Environment," Int. J. Humanoid Robot., vol. 11, no. 01, p. 1450004, 2014.

[17] S. Beyhan, R. Babu`, M. Wisse, and M. Alc1, "Adaptive Fuzzy and Sliding-Mode Control of a Robot Manipulator with Varying Payload," 15th IEEE Conference on Desision and Control and European Control Conference (ICD-ECC). pp. 8291-8296, 2011.

[18] A. B. Sharkawy, "An Adaptive Fuzzy Sliding Mode Control Scheme for Robotic Systems," Intell. Control Autom., vol. 02, no. 04, pp. 299-309, 2011.

[19] S. Głowiński, T. Krzyżyński, S. Pecolt, and I. Maciejewski, "Design of motion trajectory of an arm exoskeleton,” Arch. Appl. Mech., Aug. 2014.

[20] "https://grabcad.com/home." [Online]. Available: https://grabcad.com/home. [Accessed: 04-Nov-2015]. 